\title{
Diagnostic potential of the Ca II $8542 \AA$ line for solar filaments
}

\author{
C. J. Díaz Baso ${ }^{1,2,3}$, M. J. Martínez González ${ }^{1,2}$, A. Asensio Ramos ${ }^{1,2}$, and J. de la Cruz Rodríguez ${ }^{3}$ \\ 1 Instituto de Astrofísica de Canarias, C/Vía Láctea s/n, 38205 La Laguna, Tenerife, Spain \\ e-mail: carlos.diaz@astro.su.se \\ 2 Departamento de Astrofísica, Universidad de La Laguna, 38206 La Laguna, Tenerife, Spain \\ 3 Institute for Solar Physics, Dept. of Astronomy, Stockholm University, AlbaNova University Centre, 10691 Stockholm, Sweden
}

Received 6 December 2018 / Accepted 14 February 2019

\begin{abstract}
Aims. In this study we explore the diagnostic potential of the chromospheric Ca II line at $8542 \AA$ for studying the magnetic and dynamic properties of solar filaments. We have acquired high spatial resolution spectropolarimetric observations in the Ca II $8542 \AA$ line using the CRISP instrument at the Swedish $1 \mathrm{~m}$ Solar Telescope.

Methods. We used the NICOLE inversion code to infer physical properties from observations of a solar filament. We discuss the validity of the results due to the assumption of hydrostatic equilibrium. We have used observations from other telescopes such as CHROTEL and SDO, in order to study large scale dynamics and the long term evolution of the filament.

Results. We show that the Ca II $8542 \AA$ line encodes information of the temperature, line-of-sight velocity and magnetic field vector from the region where the filament is located. The current noise levels only allow us to estimate an upper limit of $260 \mathrm{G}$ for the total magnetic field of the filament. Our study also reveals that if we consider information from the aforementioned spectral line alone, the geometric height, the temperature and the density could be degenerated parameters outside the hydrostatic equilibrium approach.
\end{abstract}

Key words. Sun: filaments, prominences - Sun: chromosphere - Sun: magnetic fields - Sun: oscillations - Sun: evolution

\section{Introduction}

Solar filaments are cool plasma overdensities suspended at chromospheric and coronal heights. Instead of falling to the surface due to gravitational acceleration, they can remain suspended from hours to several weeks. Forces derived from the presence of magnetic fields are expected to provide the required support to form filaments. However, the exact magnetic topology of filaments and its connectivity with the photosphere are still an ongoing debate. Filament plasma is supported where the magnetic field lines are curved upwards, forming dips. The latter could be induced by the prominence plasma itself (Kippenhahn \& Schlüter 1957), or can be present in untwisted (Antiochos et al. 1994), twisted (van Ballegooijen \& Martens 1989), or tangled (van Ballegooijen \& Cranmer 2010) magnetic fields.

This controversy about the magnetic field topology is also connected with the dynamics of the filament. For example, streaming and counter-streaming flows have been identified in the spine and barbs of filaments interpreted as plasma flows along not dipped magnetic flux tubes (Zirker et al. 1998; Lin et al. 2003; Diercke et al. 2018). However, other authors, such as Heinzel (2007), show that analyzing 2D images can lead to misinterpretations because of the lack of a fully 3D dynamical pattern and the counter-streaming could be explained with oscillations rather than flows along threads.

Although many studies and simulations have been carried out in the past decades (Aulanier \& Démoulin 2003; Lites 2005; Khomenko et al. 2014; Keppens \& Xia 2014; Luna et al. 2015; Okamoto et al. 2016), new high-resolution observations are opening up the possibility of a better understanding of this solar phenomenon (Martínez González et al. 2015; Kuckein et al. 2016). These chromospheric structures can be observed in the core of strong lines with sufficient opacity to be sensitive to the physical conditions above the photosphere. Most studies have used observations acquired in $\mathrm{H} \alpha$ (Kuckein et al. 2016), the $\mathrm{He}$ I multiplets at $10830 \AA$ (Kuckein et al. 2012; Orozco Suárez et al. 2014) and $5876 \AA \mathrm{D}_{3}$, Leroy et al. 1977; López Ariste \& Casini 2002; Casini et al. 2003), and the extreme ultraviolet continua (Schmieder et al. 2003) to study filaments.

Solar filaments can also be observed in the Ca II infrared triplet at 8498,8542 and $8662 \AA$. The latter have relatively low effective Landé factors $\left(\bar{g}_{8498}=1.07, \bar{g}_{8542}=1.10\right.$, and $\bar{g}_{8662}=$ 0.87). Although they are not especially sensitive to the presence of magnetic fields, these lines can be observed over the entire solar disk (as opposite to the He I $10830 \AA$ triplet, whose opacity in the quiet Sun is extremely low) and we can use them to extract information about the plasma temperature (Socas-Navarro et al. 2000). Non-local thermodynamic equilibrium (NLTE) radiative transfer is required to model the $\mathrm{Ca}$ II IR lines, in this case the assumption of statistical equilibrium and complete frequency and angle redistribution suffice to retrieve accurate results (Uitenbroek 1989; Wedemeyer-Böhm \& Carlsson 2011).

The Ca II $8542 \AA$ is the line most commonly used in previous studies because it has the largest opacity among the triplet lines. Additionally, it has the largest Landé factor of the triplet and it is not blended as the Ca II $8498 \AA$ line is. The Ca II IR lines are very sensitive to the temperature stratification of the lower chromosphere and 3D radiative transfer effects can be neglected (de la Cruz Rodríguez et al. 2012; Leenaarts \& Carlsson 2009; Štěpán \& Trujillo Bueno 2016). Despite these advantages, its low polarimetric sensitivity has limited the study of magnetic fields to active regions with large magnetic field strengths (Socas-Navarro et al. 2000; Socas-Navarro 2005; de la Cruz Rodríguez et al. 2013a, 2015a; Asensio Ramos et al. 2017). 
Outside the active regions, the magnetic field is so weak that scattering polarization and the Hanle effect must be included,especially in observations acquired toward the limb. The Hanle regime of the Ca II $8542 \AA$ line is between $0.001<$ $B<0.1 \mathrm{G}$ and the Hanle saturation regime for $B>10 \mathrm{G}$ (Manso Sainz \& Trujillo Bueno 2010). Above $100 \mathrm{G}$ the line is again sensitive to the magnetic field vector through the Zeeman effect. However, the presence of 3D and dynamic effects in the radiative transfer problem make it very computationally time consuming (Carlin et al. 2013; Štěpán \& Trujillo Bueno 2016).

There are only a few studies of prominences (off-limb filaments) in the Ca II $8542 \AA$ line (Stellmacher et al. 2003; Park et al. 2013) where they calculate the temperature from the line broadening using two lines of different atoms to separate the contribution of the thermal and non-thermal motions, using $\mathrm{He}_{\mathrm{I}} 10830 \AA, \mathrm{H} \alpha$, and $\mathrm{H} \beta$. For filaments (on-disk) the background, which is illuminating the structure, needs to be taken into account and cloud models are used (see the review by Tziotziou \& Heinzel 2007).

In this study we present the analysis of very high spatial resolution spectropolarimetric observations acquired in the $\mathrm{Ca}$ II $8542 \AA$ line which are analyzed using the NLTE inversion code NICOLE (Socas-Navarro et al. 2000, 2015). In our analysis we have explored the potential diagnostic of the $\mathrm{Ca}$ II line and we discuss the validity of inversion results due to the assumption of hydrostatic equilibrium. To study the global dynamics of the region and the evolution of the filament, we used observations from other synoptic telescopes.

\section{Observations}

The observations analyzed in this study were acquired at the Swedish $1 \mathrm{~m}$ Solar Telescope (SST; Scharmer et al. 2003) on the 22nd of July 2013 using the CRISP imaging spectropolarimeter (Scharmer et al. 2008). The telescope was pointed to a solar filament situated far from disk center at coordinates $x, y=(+653,-305)^{\prime \prime}$, which corresponds to heliocentric distance $\mu=\cos \theta=0.65$.

The Ca II $8542 \AA$ line was sampled in the range $\pm 1.75 \AA$ from the core in 21 line positions sampled with varying step size: $70 \mathrm{~m} \AA$ close to the core of the line, and up to $800 \mathrm{~m} \AA$ in the far wings (see lower panels of Fig. 1). This strategy (de la Cruz Rodríguez et al. 2012, 2013a) is used to optimize the time cadence of the observations by placing less spectral points in the far photospheric wings. The observations consist of 36 scans taken with a cadence of $\sim 23.22 \mathrm{~s}$. Simultaneous observations in the $\mathrm{Fe}_{\mathrm{I}} 6301 / 6302 \AA$ lines were also acquired but not used in the present study.

Figure 1 shows the field of view (FOV) at different wavelength positions. We have chosen three different $\lambda-\lambda_{0}$ offsets at $-0.945,-0.28$, and $0.0 \AA$ from the line center in order to visualize our mapping of different atmospheric regimes as we move across the spectral line. In the left panel, the image depicts reversed granulation at a height of around $140 \mathrm{~km}$ (Cheung et al. 2007) where the intergranular lanes are relatively hotter than the granules, in contrast to what is observed at the lower photosphere. This image gives us an idea of which are the deepest layers we can measure given that we do not reach continuum wavelengths. The middle panel shows a filamentary structure that covers the whole FOV. This structure is even more enhanced in the right panel, where bright plage areas are surrounding the dark filament showed in absorption in the core of the Ca II $8542 \AA$ line. This filament is the focus of this study.

\subsection{Data reduction process}

The reduction of the CRISP dataset was carried out using the CRISPRED pipeline (de la Cruz Rodríguez et al. 2015b). This software takes into account the dark current subtraction, flatfield correction, and demodulation process. The data have been processed with the MOMFBD code (van Noort et al. 2005) to remove blurring effects of atmospheric distortion while bringing the spatial resolution close to the diffraction limit of the telescope at this wavelength $\left(\sim 0.18^{\prime \prime}\right)$. The resulting noise level is in the range $4-6 \times 10^{-3}$ in units of the continuum intensity for Stokes $Q, U$, and $V$.

After the standard data reduction, a final inspection showed some residual artifacts that needed to be corrected. The first is the presence of polarized interference fringes. These fringes appear in the individual monochromatic images. The spatial pattern of these fringes is different for each Stokes parameter and it is also wavelength dependent. To correct these fringes we computed Fourier filters for each individual wavelength and Stokes parameter. The frequency amplitude of these fringes is well localized, far away from the frequencies involved in the data and a threshold is used to set those peak coefficients to zero (i.e., we did not use a rectangular or fixed shape to mask the fringe frequencies). Then, we performed the inverse Fourier transform to recover our clean image. We note that these fringes are almost at the level of our signals and a very careful estimation of the fringe pattern is required to properly retrieve the magnetic field vector. After the cleaning process, though not completely removed, the presence of fringes was strongly alleviated.

For the correction of very-long period fringes or cross-talk, we have used monochromatic images at continuum wavelengths of each Stokes parameter. The advantage of continuum wavelengths (or very far wings in this case) is that we do not expect any significant structure in Stokes $Q, U$, or $V$. Therefore, the identification of this effect is easier and more accurate. In order to apply this correction, we assumed that every monochromatic image for each Stokes parameter is composed of the true measurement contaminated by a quantity that is proportional to the signal detected in the line wing:

$X(\lambda)=X_{0}(\lambda)+C_{\mathrm{X}, \lambda} X_{\mathrm{W}}$.

The coefficient $C_{\mathrm{X}, \lambda}$ is estimated by minimizing the absolute correlation between the far-wing image and each monochromatic image, and this contribution is then removed from the input image. Since measurements at different wavelengths on the continuum show slightly different patterns, we cannot ensure the accuracy of the polarization signals below or at the level of $6 \times 10^{-4} I_{\mathrm{c}}$ as the artifacts have an amplitude of the same order.

Finally, to normalize the spectrum by the continuum intensity we used the Fourier Transform Spectrometer $\left(I_{\mathrm{FTS}}(\lambda)\right.$ or FTS, Neckel \& Labs 1984) atlas as a reference. Since the FTS is taken at disk center, we compared it with a spectrum taken at the same position (which we call $I_{0}$ ) a few hours before the observations that we wanted to analyze (referred to as $I_{1}$ ), taken at other disk position (e.g., $\mu=0.65$ ). The average of a quiet-Sun region of the disk center observation is compared with the FTS atlas after convolving with the instrument PSF, $\sigma \sim 108 \mathrm{m \AA}$ :

$\left\langle I_{\mathrm{QS} 0}\right\rangle \simeq I_{\mathrm{C} 0} \cdot\left[I_{\mathrm{FTS}} * G(\sigma)\right]$.

We then needed to compensate for changes in the observed intensities due to variations in the airmass during the observations, and take into account the ratio of the center-tolimb variation of the continuum intensity, i.e., $R_{\lambda \mu}=I_{\mathrm{C}}(\lambda=$ 

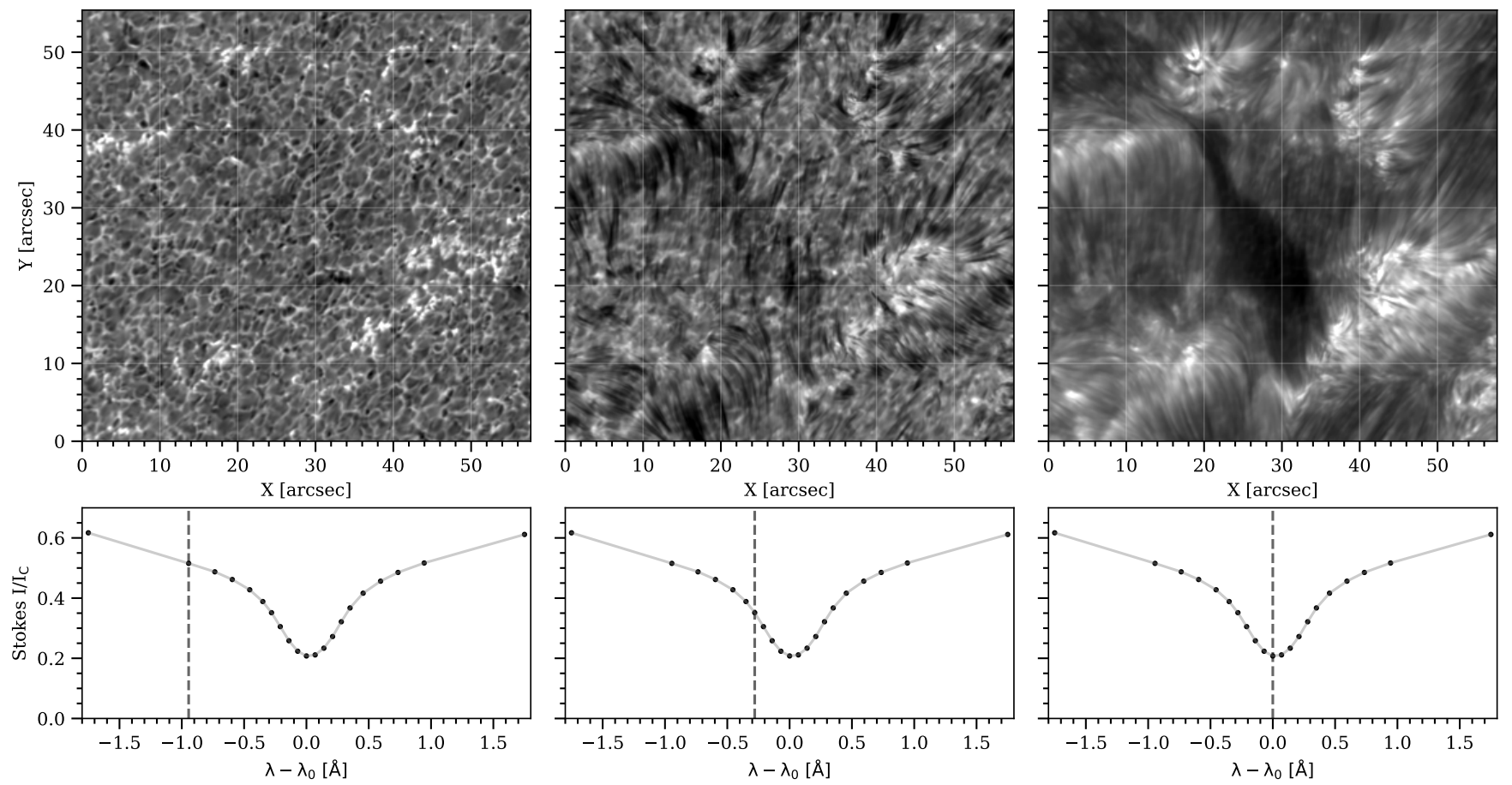

Fig. 1. Field of view at three different wavelength positions $-0.945,-0.28$, and $0.0 \AA$ from the line center $\lambda_{0}=8542.1 \AA$. Lower panels: average line profile and vertical dashed line at the wavelength used to generate the FOV of the upper panel. We see how the spectral line has information from the photosphere (left column) to the chromosphere (right column) where the filament is observed.

$8542.0, \mu=0.65) / I_{\mathrm{C}}(\lambda=8542.0, \mu=1.0)$. This ratio is calculated from the values tabulated in Cox (2000). The new quietSun continuum is:

$I_{\mathrm{C} 1}=I_{\mathrm{C} 0} \cdot I_{\mathrm{WB} 1} /\left(I_{\mathrm{WB} 0} \cdot R_{\lambda \mu}\right)$,

where $I_{\mathrm{WB}}$ are the average intensity of the broadband images of each observation. After this, all that remained was to divide all the profiles by this quantity.

\subsection{Solar context}

To put the observations in context, we have made use of data provided by some of the band-pass filters (AIA; Lemen et al. 2012) on board NASA's Solar Dynamics Observatory (SDO; Pesnell et al. 2012), by the Global Oscillation Network Group (GONG; Hill et al. 1994) and from the Chromospheric Telescope (CHROTEL; Bethge et al. 2011). We display in Fig. 2 images taken at a time in the middle of the observations (around 09:24 UT). The top left panel of Fig. 2 shows an SDO/HMI image at the continuum of $6173 \AA$ of the region where we can see the quiet Sun (QS) below our filament.

The rest of panels of Fig. 2 show wavelengths sensitive to higher temperatures at chromospheric and coronal heights. Despite the lower resolution of these images $\left(\sim 1.2^{\prime \prime} \mathrm{pix}^{-1}\right.$ in HMI, $\sim 1.6^{\prime \prime} \mathrm{pix}^{-1}$ in AIA, and $\sim 5^{\prime \prime} \mathrm{pix}^{-1}$ in GONG) the filament is more visible in the filters sensitive to lower temperatures such as He II $304 \AA$, while less visible in the coronal filters Fe IX $171 \AA$, Fe XII $193 \AA$, and Fe xIV $211 \AA$. Other filters sensitive to larger temperatures (like Fe xvIII $94 \AA$ ) do not show any hint of the filament. Finally, the filament becomes clearly visible with a high absorption in the $\mathrm{H} \alpha$ filter of GONG and the narrow filter of He I of CHROTEL (lower right panels).

Thanks to the temporal cadence of GONG and SDO, we can trace the evolution of the filament from its formation until it disappears behind the limb. Figure 3 shows the evolution of the filament for five days. Throughout the evolution we see how the filament is increasing its absorption (it becomes darker) and its size. The top row shows the filament in $\mathrm{H} \alpha$ taken from the GONG network, where the first four images are from GONGTeide which has a better resolution, and the last one is from GONG-Mauna Loa. The second row shows the same area in the AIA/171 $\AA$ filter, but large coronal loops in the region mask the filament. Our spectropolarimetric observation was made two days after its formation (third column). Between the third and fourth day the filament starts to decay and it almost vanishes. A few hours later, the body starts to acquire absorption again. The last column shows the filament at the limb, where a small bright cloud appears to protrude by almost $25^{\prime \prime}$ above the limb. However, due to all the activity of the area, we cannot be sure that the filament seen in this frame is the same as that of our observation, as the physical properties of the region could have changed significantly in this period of time.

\section{Data analysis}

After the complete reduction process, including fringes and crosstalk correction, we prepared the data to be analyzed with the NICOLE code. To increase the signal-to-noise ratio of our observations, we average the entire time series since we see no substantial changes in the evolution of the filament. Additionally, we also downsampled the image with a binning of $4 \times 4$ pixels, yielding a final sampling of $0.24^{\prime \prime}$ pix $^{-1}$. We clarify that all this processing is not really needed for Stokes $I$, but it becomes necessary for the polarization to significantly improve the signal-to-noise ratio. We decrease the photon noise from $4-6 \times 10^{-3}$ to $2-6 \times 10^{-4}$ in units of the continuum intensity for Stokes $Q, U$, and $V$. Despite the high polarimetric sensitivity, some systematic artifacts are still present. We discuss these in the following sections. 



Fig. 2. Area of $100^{\prime \prime} \times 100^{\prime \prime}$, with a square displaying our FOV. The wavelength range at which each map is taken is indicated at the top of each panel. The axis scales have their origin at the disk center, being the vertical the N-S direction while the horizontal the W-E direction, with negative sign toward the south and the west.
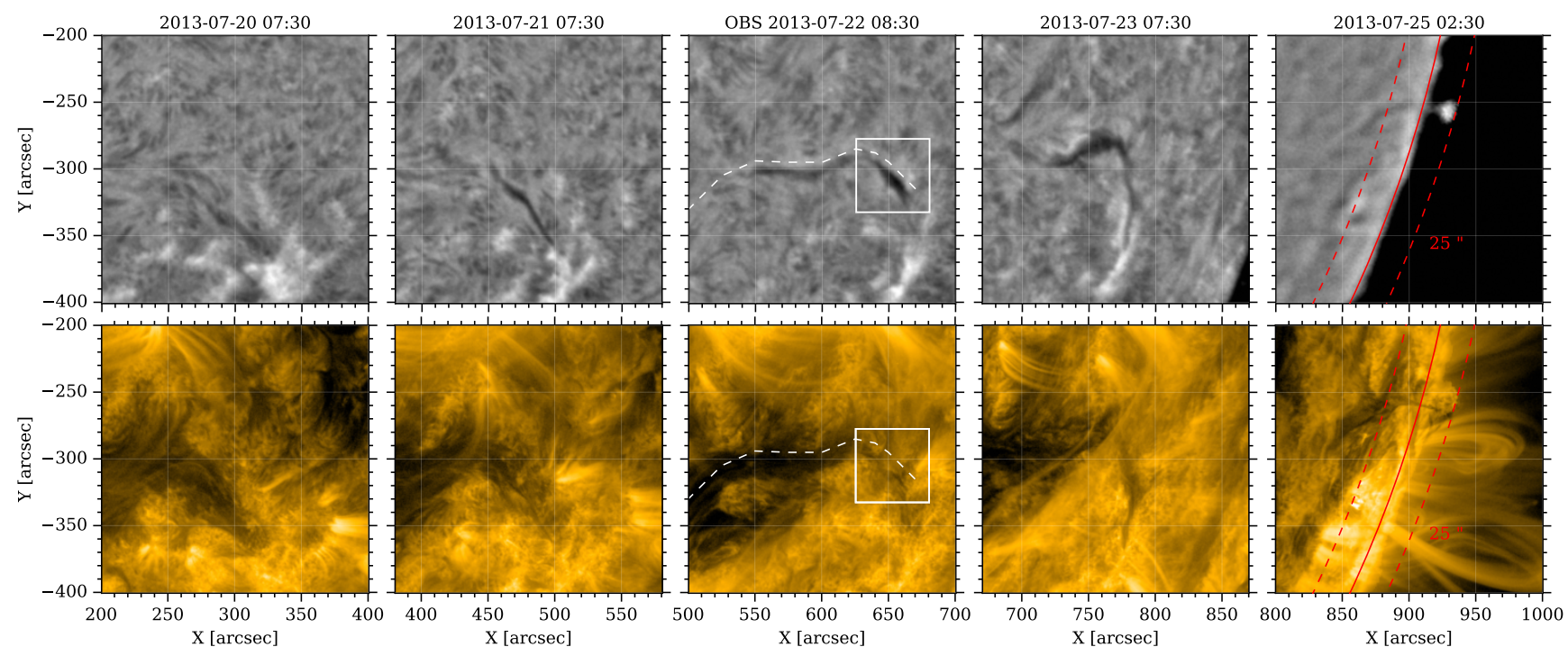

Fig. 3. Evolution of the filament during five days. We see how the filament is increasing its absorption and its size (up to $100^{\prime \prime}$ long) during its evolution. Top row: images from GONG and bottom row: same area but in the channel $171 \AA$ of AIA. A dashed white line indicates the shape of the whole structure visually estimated from the combination of both filters.

\subsection{Inversion process}

We use the NICOLE inversion code (Socas-Navarro et al. 2000, 2015) to infer the physical conditions in the lower solar chromosphere. Like other inversion codes (e.g., SIR, Ruiz Cobo \& del Toro Iniesta 1992), NICOLE iteratively perturbs the physical parameters of a model atmosphere such as the temperature, lineof-sight velocity, and magnetic field to find a synthetic spectra that can reproduce the observations. The stratification of density and gas pressure are computed by assuming hydrostatic equilibrium (HE). The physical parameters of the resulting model atmosphere are given as functions of the optical depth scale at $5000 \AA ̊ \cap, \log (\tau)$. We have used a Ca II model atom consisting of five bound levels plus continuum which is synthesized assuming complete frequency redistribution (CRD) and including the effect of Ca II isotopic splitting (Leenaarts et al. 2014).

We have used the spectral transmission profile of CRISP to degrade the emerging Stokes profiles during the inversion. According to de la Cruz Rodríguez et al. (2015b), the FWHM in this spectral range is $\sim 108 \mathrm{~m} \AA$ and the Nyquist-Shannon (NS) sampling theorem establishes that the sampling has to be at least $54 \mathrm{~m} \AA$. As the observations were taken with a minimum sampling of $70 \mathrm{~m} \AA$, we synthesized the profiles with a wavelength axis with a sampling of $35 \mathrm{~m} \AA$. This new grid contains exactly all the observed points and fulfills the NS theorem. Moreover some 

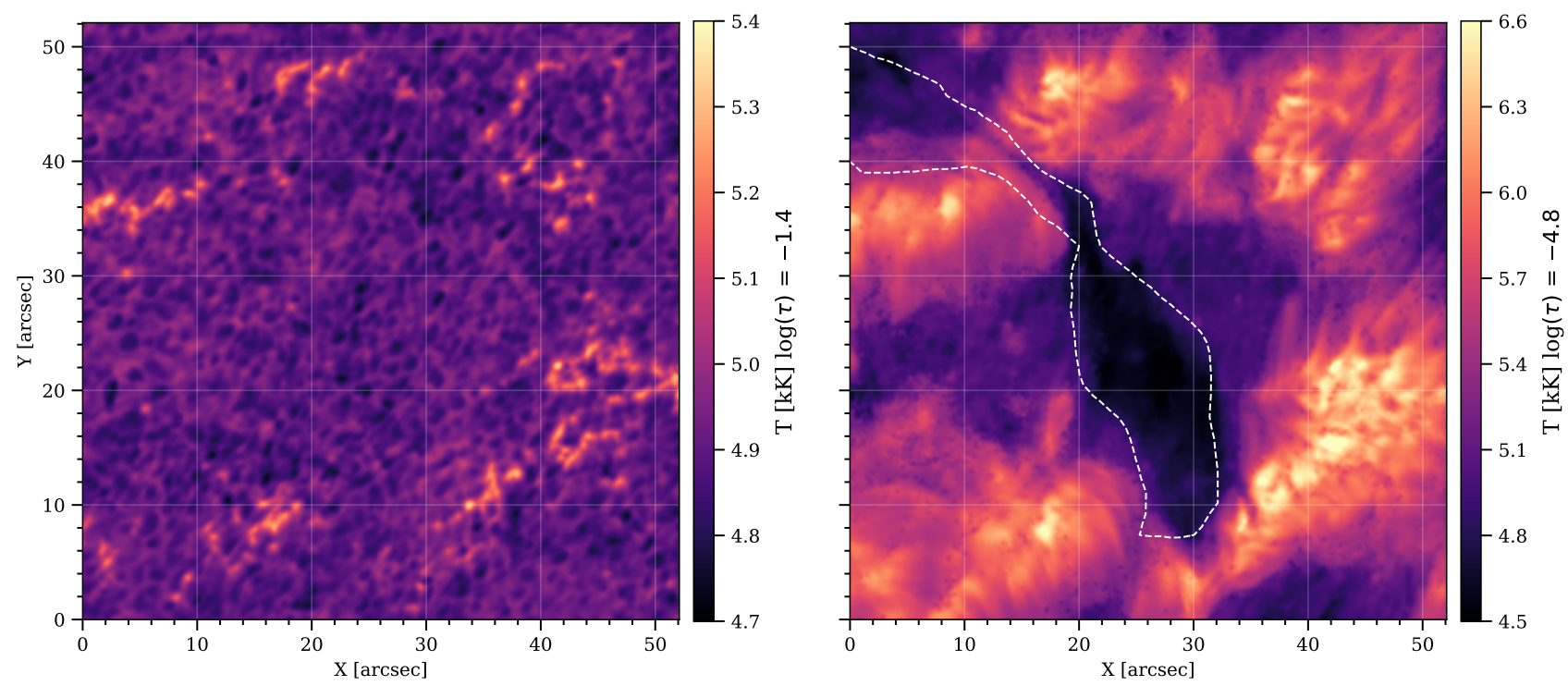

Fig. 4. Temperature stratification inferred with NICOLE at $\log (\tau)=-1.4$ (left panel) and $\log (\tau)=-4.8$ (right panel). The shape of the filament seen in $\mathrm{H} \alpha$ by СнroтеL is indicated by the white dashed contour.

points are added in the border of the wavelength range to avoid artifacts during the convolution. In a final step, our observed profiles are interpolated to the new grid but only the observed points are compared with the synthetic ones. We accomplished that by only weighting these points accordingly in the merit function.

In order to reduce the complexity of the problem and speed up the inversion, we decided to fix the microturbulent velocity. We have inverted several pixels across the FOV including different solar structures with the microturbulent velocity as a free parameter and we estimated an average value of $3 \mathrm{~km} \mathrm{~s}^{-1}$ with a small dispersion of $\sim 1 \mathrm{~km} \mathrm{~s}^{-1}$. Microturbulence is often associated with line broadening produced by unresolved plasma motions. However, we want to stress that it can be slightly degenerated to the temperature unless different spectral lines of different atoms are available. As we see below, the average profiles of different regions (shown in the right panel of Fig. 5) have a similar width, and their main difference is their depth. Although the absolute values of temperature could be slightly affected, the main conclusions of the paper would remain unaffected. We have also verified that the average Stokes profiles of the time series have the same broadening as that of individual profiles, meaning that the time scale of the filament evolution is larger than the duration of our series.

We have used the default equation of state (EOS) in NICOLE to perform the full-FOV inversions. Concerning the upper boundary conditions for the gas pressure for the HE inversions we have used the same value of $0.3 \mathrm{dyn} \mathrm{cm}^{-2}$ for all pixels. In the following, when pressure is mentioned, we are always referring to the total gas pressure. The inversions are done pixel by pixel, in other words, the NLTE problem is solved for each pixel independently assuming a plane-parallel atmosphere. This approximation is valid for the Ca II $8542 \AA$ line where horizontal photon scattering can be neglected (de la Cruz Rodríguez et al. 2012) ${ }^{1}$. To improve the convergence of our inversions, we performed three cycles. In the first cycle, we chose four nodes equidistantly located in the $\log (\tau)$ axis for temperature, one in line-of-sight (LOS) velocity and one more in each one of the three components of the magnetic field. This first cycle is very robust as there are no correlations between the magnitudes: the

1 See Štěpán \& Trujillo Bueno (2016) for the limits of this assumption. temperature describes the depth and width of the line, one node in LOS velocity mostly affects the core of the line (given the response function of the spectral line to the velocity and the dense sampling in the core), and the magnetic field describes the signal in Stokes $Q, U$, and $V$. Given the weak nature of the magnetic field strengths that are involved in our study, the Ca II $8542 \AA$ line is in the weak field Zeeman regime and the Stokes $I$ is very weakly affected by the Zeeman broadening. The initial guess model is sampled with 43 points from $\log (\tau)=-7.2$ to $\log (\tau)=+1.2$. We started with a temperature stratification with an almost arbitrary parabolic shape as we do not need a better initial model to fit the spectral line. This robust first cycle is able to capture the main information and model the line profile with excellent convergence. To reduce possible artifacts, a horizontal smooth (in $\log (\tau)$ scale) is applied after each cycle. We used a median filter with a kernel size of three pixels to remove bad fits and a Gaussian filter with a FWHM of three pixels to smooth the whole FOV. This filter is applied at the same node positions of the inversion and then a Bezier-spline interpolation is applied (de la Cruz Rodríguez \& Piskunov 2013). The output of this smoothing process constitutes the input model for the next cycle. As strong asymmetries are not detected by an inspection of Stokes profiles and the FOV seems to be dynamically quite stable, only a maximum of two nodes are set to the LOS velocity in the second cycle. This is enough to capture the different velocities at the photospheric and chromospheric levels. A similar reasoning is applied to the LOS component of the magnetic field, as some Stokes $V$ profiles show several lobes indicating gradients of magnetic fields along the LOS. A final cycle is needed to improve the model of complex profiles located in the plage region. In this final cycle, the number of nodes in temperature is increased to seven, in other words, we added one node between each pair of nodes from the previous cycle. The inversion strategy used to invert the full FOV is shown in Table 1.

\subsection{Temperature stratification}

To visualize the temperature stratification we have taken two horizontal slices at two different optical depths which are shown in Fig. 4: at $\log (\tau)=-1.4$, associated to a photospheric layer, and at $\log (\tau)=-4.8$, which provides a chromospheric view. These 

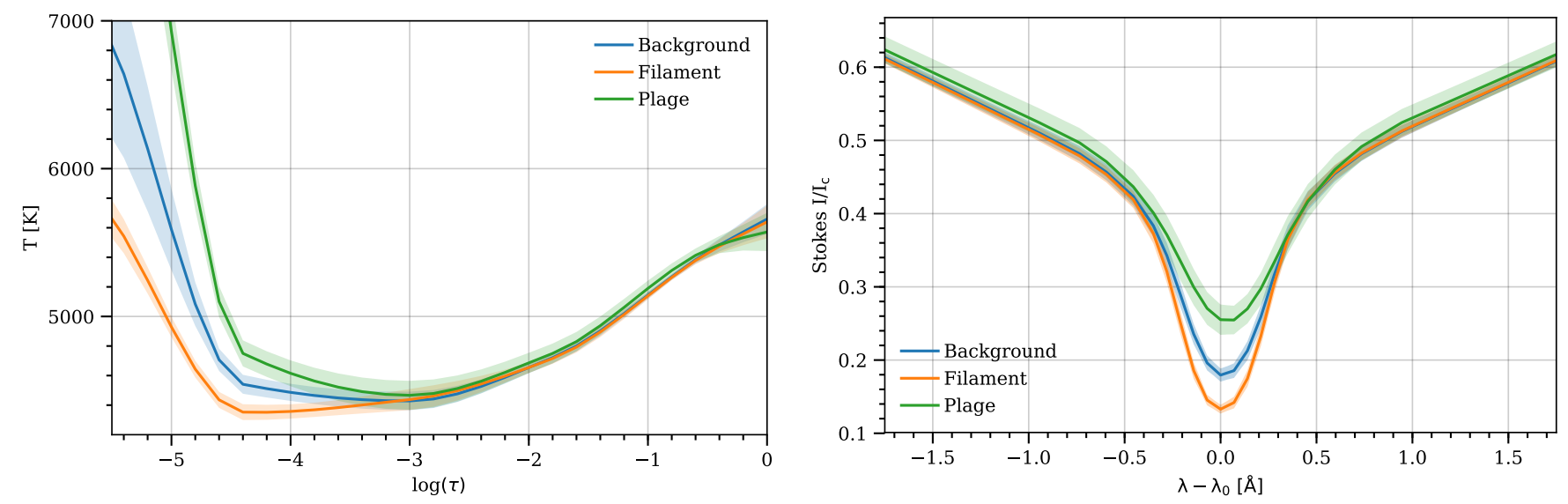

Fig. 5. Left panel: temperature stratification calculated from the average of the temperature inferred from profiles of different regions. Right panel: average Stokes I profile of the selected regions. The colored bands of each curve indicate the standard deviation of each set of profiles.

Table 1. Nodes used to invert each pixel with the inversion code NICOLE.

\begin{tabular}{ccccc}
\hline \hline Cycle & Temperature & $v_{\text {LOS }}$ & $B_{\text {LOS }}$ & $B_{\perp}$ \\
\hline First & 4 nodes & 1 node & 1 node & 1 node \\
Second & 4 nodes & 2 nodes & 2 nodes & 1 node \\
Third & 7 nodes & 2 nodes & 2 nodes & 1 node \\
\hline
\end{tabular}

Notes. Microturbulent and macroturbulent velocities are not inverted.

values have been chosen at two very sensitive layers of the 8542 line in quiet Sun conditions (Quintero Noda et al. 2016). The left panel in Fig. 4 depicts a very similar picture to that in the left panel in Fig. 1, that is, a photospheric reversed granulation pattern with temperatures of about $5000 \mathrm{~K}$. The second panel shows a cold filament with an average temperature of $4500 \mathrm{~K}$, surrounded by hot plage regions with a temperatures larger than $6500 \mathrm{~K}$.

To better study the observed area, we have classified the spectral profiles in three different classes according to the intensity in the core of the line. Filament profiles are those with intensities less than $0.148 I_{\mathrm{c}}{ }^{2}$, plage profiles have intensities higher than $0.22 I_{\mathrm{c}}$ and background profiles are those with intensities between the two. The left panel of Fig. 5 shows the average temperature stratification of each type of profile, the green line showing the temperature of the plage, the blue line displaying the temperature of the background and the orange line showing the temperature of the filament. We have extended the axis only to $\log (\tau)=-5.5$ because the line becomes insensitive after that point (Quintero Noda et al. 2016). This figure demonstrates that the three curves overlap below $\log (\tau)=-3$ and start to separate above. At $\log (\tau)=-5.5$ the difference between the filament and the environment is of around $1000 \mathrm{~K}$. The right panel of Fig. 5 displays the average profiles for each type. The shaded bands around the curves indicate the standard deviation of each set of profiles (in both panels).

\subsection{Longitudinal magnetic field}

We focus now on the longitudinal component of the magnetic field as inferred by NICOLE. We show this quantity at two

\footnotetext{
2 This number has been chosen by inspection to enclose the shape of the filament. The same applies to the plage threshold.
}

heights in Fig. 6. As expected, the Stokes $V$ parameter encodes information from the photosphere to the chromosphere and the inversion process is able to extract gradients of the LOS magnetic field with height.

In the photosphere (left panel of Fig. 6) the magnetic field appears more concentrated at small scales, while it is more diffuse in the chromosphere (right panel of the same figure), though maintaining the general topology of the photospheric field. The reason is that as the pressure decreases, the magnetic field expands. Probably, the magnetic field in the filament area appears uniform because we are tracing the upper part of the canopy, while the small loops are closing at lower heights. Although some small patches of opposite polarity can be seen in the photosphere (as well in the wings of Stokes $V$ ), we cannot ensure that these points do, in fact, have different polarities. They are usually close to the plage and hotter than the rest of the FOV. In such cases, two peaks at both sides of the core of the line usually appear in emission (in Stokes $I$ ) generating a Stokes $V$ signal with a different sign in the wings. This can be incorrectly interpreted by the inversion code as a reversal in the magnetic field. The FOV has a plage of almost $1 \mathrm{kG}$ in the photosphere that decreases up to $500 \mathrm{G}$ in the chromosphere, with a very weak magnetic field in the middle where the filament is located. One would expect to see the filament in these maps if the dense filament plasma is modifying the magnetic field lines of the region. However, there is no clear indication of its presence as we found similar values inside and outside the filament, unlike its clear detection in the temperature map. From this magnetic field map, it is difficult to distinguish whether the filament is simply embedded in the lower atmosphere of the region with a similar magnetic field, or with a weaker magnetic field well above the solar surface and thus not detected.

\subsection{Transverse magnetic field}

We have used only one node to infer the transversal component of the magnetic field. It means that the inferred stratification is constant, with the same value at all the heights. The map of $B_{\perp}$ is shown in the left panel of the Fig. 7. This map shows higher values in the plage, while the filament is permeated by a very weak transverse magnetic field of less than $200 \mathrm{G}$.

The linear polarization signals detected in the region are rather weak, on the order of $10^{-4} I_{\mathrm{c}}$ in most of the map. We show the line core linear polarization in the left panel of Fig. 7 where only some very small patches show signals above $8 \times 10^{-4} I_{\mathrm{c}}$. 

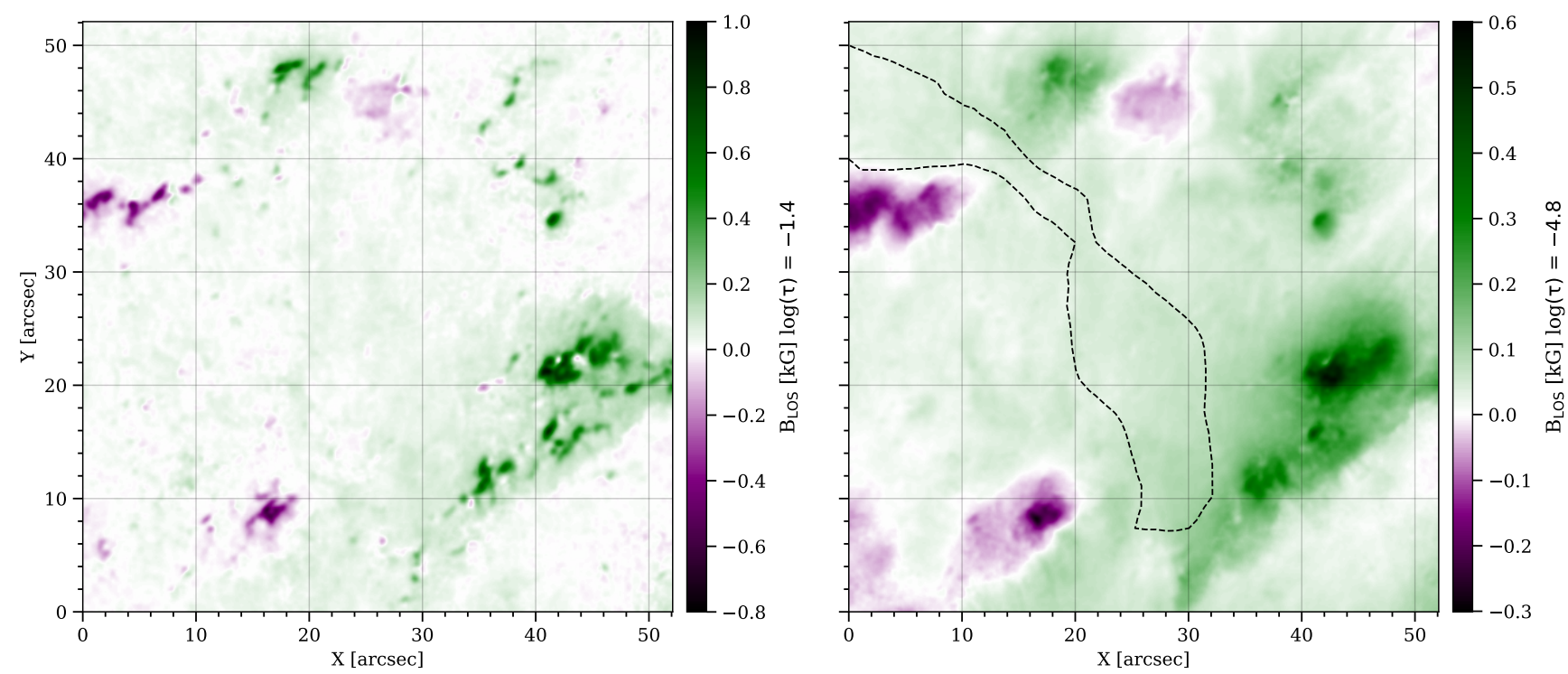

Fig. 6. Longitudinal component of the magnetic field inferred with NICOLE at two levels: the $\log (\tau)=-1.4($ left panel $)$ and $\log (\tau)=-4.8($ right panel).

We note that the residual systematic polarization artifacts that are present in our observations can strongly affect the observed Stokes profiles at the achieved $\mathrm{S} / \mathrm{N}$ level. The latter are well above the photon noise and they do not decrease as we integrate a longer time series. An example of this effect is displayed in Fig. 8, which shows the Stokes profiles at position $\left(25^{\prime \prime}, 25^{\prime \prime}\right)$ inside the filament. This figure shows that the fit is properly done. Stokes $Q$ and $U$ do not have enough signal, so that the inversion can fluctuate provided that the profiles produce similar merit function values. This poses some doubts about the uncertainty of the inferred transverse magnetic field.

Martínez González et al. (2012) studied the effect of the noise in the estimation of the azimuth $\Phi_{B}$, inclination $\Theta_{B}$, longitudinal magnetic field $B_{\|}$, and transverse magnetic field $B_{\perp}$ in the weak field approximation. They found that the maximum likelihood estimation of the longitudinal component and the azimuthal angle are unbiased quantities. On the contrary, the transverse component, and hence the inclination angle, presents a non-zero bias, i.e., there is a difference between the value of the estimator and the true value of the parameter due to the presence of noise. The value of the bias is derived by Martínez González et al. (2012) and it is given by

$B_{\perp}^{\mathrm{bias}}(\sigma)=\left(\frac{-2 \ln \mathrm{c}}{\sum_{j} I_{j}^{\prime \prime 2}}\right)^{1 / 4} \frac{\sqrt{\sigma}}{C}$,

where $C=4.67 .10^{-13} \lambda_{0}^{2} \sqrt{G} / 2, G=1.22$, and $\lambda_{0}=8542 \AA$ in the case of this line. If the inferred $B_{\perp}$ is similar or smaller than the bias, this value may not be correct and may be strongly affected by the noise of the observations. Using the percentile $50(c=0.5)$ and a very conservative value for the photon noise of $\sigma=10^{-4} I_{\mathrm{c}}$, we obtain the map of bias displayed in the right panel of Fig. 7. The bias is proportional to the noise standard deviation so that, a lower value generates a lower bias and thus more reliable values of $B_{\perp}$.

We have drawn contours at the values of the bias in the left panel of Fig. 7 indicating the locations in which the inferred value is above the bias. The contours show that there are only small areas where the inferred field is well above this bias and almost the rest of the map is below it. Therefore, the inferred value of $B_{\perp}$ is dominated by the noise in large part of the FOV, something that is expected given that Stokes $Q$ and $U$ have low polarization signals in the area. The spatial pattern of the bias, with low values in the center of the map and higher values in the border of the map coinciding with the plage, is a consequence of the line depth. The dependence of Eq. (4) on the second derivative of the intensity profile produces a larger bias for shallower lines. Therefore, we can give only an upper limit on the transversal magnetic field of the filament of around $250 \mathrm{G}$. Using the same formula, we can also estimate the noise required to have a bias below a certain magnetic field. For instance, for a magnetic field of $B_{\perp}=200 \mathrm{G}$, we estimate that one needs a photon noise below $\sigma=5 \times 10^{-5} I_{\mathrm{c}}$ for a proper inference ${ }^{3}$.

\subsection{LOS velocity}

Figure 9 shows the LOS velocity at $\log (\tau)=-1.4$ and $\log (\tau)=$ -4.8 . After the inversion an offset was added to the whole velocity stratification to end up with the photosphere with zero mean velocity ${ }^{4}$. The left panel shows small velocities across the FOV, with the typical granulation pattern with upflows in the granules and downflows in the intergranular lanes.

The right panel of Fig. 9 shows the velocity map at the optical depth where we see the imprint of the filament also in temperature. Fast downflows of almost $10 \mathrm{~km} \mathrm{~s}^{-1}$ are found in the plage, while the area of the filament is almost at rest (with respect to the photosphere). A strong upflow of almost $5 \mathrm{~km} \mathrm{~s}^{-1}$ is found at $\left(15^{\prime \prime}, 40^{\prime \prime}\right)$, just where the "tail" of the filament is located.

\footnotetext{
3 This value is only estimated using the Zeeman effect, so if the Hanle effect is included in the calculation, this value will be slightly higher. This value also assumes that the inference is calculated through the weak field approximation and therefore may differ slightly from the uncertainty calculated with NICOLE.

4 Due to cavity errors (small imperfections of the reflecting surfaces of the etalons), the velocity map can show an artificial spatial gradient. To fix it, instead of adding a constant offset to the whole map, we have calculated a smooth version of the photospheric velocity map at $\log (\tau)=0$ by convolving the map with a Gaussian of a FWHM of 30 pixels, thus removing the fine structure of the image. These values oscillate between $-0.8 \mathrm{~km} \mathrm{~s}^{-1}$ and $+0.2 \mathrm{~km} \mathrm{~s}^{-1}$.
} 



Fig. 7. Left panel: linear polarization calculated at the core of the spectral line $\sqrt{Q\left(\lambda_{0}\right)^{2}+U\left(\lambda_{0}\right)^{2}}$. Middle panel: transversal magnetic field inferred during the inversion. Right panel: magnetic field bias generated with a noise of $\sigma=10^{-4} I_{\mathrm{c}}$. Some solid contours have been drawn in the left panel indicating where the inferred value is above the bias. The rest of values are overestimated. The white dashed contour draws the shape of the filament seen in $\mathrm{H} \alpha$ by Chrotel.
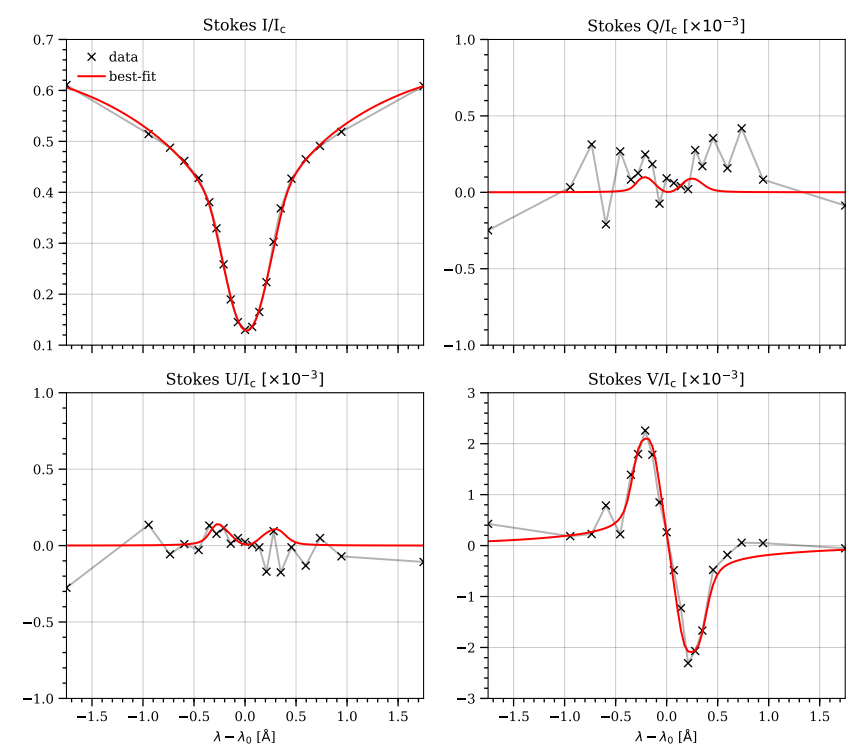

Fig. 8. A Stokes profiles sample from inside the filament $\left(25^{\prime \prime}, 25^{\prime \prime}\right)$ shows how the fit was done properly, but as Stokes $Q / I_{\mathrm{c}}$ and $U / I_{\mathrm{c}}$ do not have enough signal, the inversion code can generate whatever profile with similar merit function values. The magnetic field inferred at $\log (\tau)=-5$ is $B_{\|} \sim 60 \mathrm{G}$ and $B_{\perp} \sim 150 \mathrm{G}$.

To obtain a better global picture and confirm the veracity of the velocity pattern of the filament, we have used observations from Chrotel. It is a robotic telescope for full-disk synoptic observations at the $\mathrm{Ca}$ II $\mathrm{K}, \mathrm{H} \alpha$, and $\mathrm{He}_{\mathrm{I}} 10830 \AA$ A chromospheric lines. Chrotel uses a tunable filter for observing the $\mathrm{He}$ I line, which allows us to estimate the line-of-sight velocity. Due to the presence of several line components and the weak absorption of the multiplet, the calibration of the LOS velocity in Chrotel is difficult (Bethge et al. 2011). For this reason, we show only a Dopplergram computed from the subtraction of two filtergrams at $\pm 0.7 \AA$ in arbitrary units (see Fig. 10). The velocity pattern detected in this line is very similar to that inferred for the Ca II line, displaying upflows of $\sim 7 \mathrm{~km} \mathrm{~s}^{-1}$ in the border of the filament. Additionally, we also detected redshifted profiles with velocities of up to $\sim 5 \mathrm{~km} \mathrm{~s}^{-1}$ in the lower part of the filament. These values have been calculated from some individual profiles. This might be a consequence of neutral material (Khomenko et al. 2016) escaping from the structure because this is not detected in Ca II.
We studied the surroundings of the filament to understand the flow of material. According to the middle panel of Fig. 3, the filament seems to be the tail of a larger filament which covers the first half of the figure (indicated with a dashed white line) and could be feeding the filament with material. This flow of material could perfectly be parallel to the surface because it would have a component in the LOS direction due to the geometry.

\subsection{Transverse velocity}

A priori, it is not possible to diagnose transverse velocities with spectral lines because they produce no Doppler shift. We have used a local correlation tracking algorithm (LCT; Yi \& Molowny-Horas 1995) to analyze the transverse flows in the images. This technique computes the intensity displacement between each two consecutive images and associates the optical flow to plasma velocities. We have used the Python implementation ${ }^{5}$ of this technique by Campos Rozo \& Vargas Domínguez (2015).

The applicability of this method to the chromosphere is very delicate. Changes in the intensity due to temperature or opacity changes might be interpreted by the code as something that is moving. In addition, inside the filament, where the contrast is very low, the estimation cannot be properly done. Finally, solar atmospheric changes can affect the estimation of transverse velocities because they deform the solar images.

Despite the aforementioned limitation of the technique, we have applied the LCT method to the core images of the Ca II $8542 \AA$, where the filament is clearly visible and to the wing images at $-1.8 \AA$ from the line center. Since the filament is stable during the 14 min of the scan, we have taken the average of the flow estimated in all the frames. This allows us to cancel certain random motions or bad spurious estimations. We have used different spatial windows between $3^{\prime \prime}$ and $5^{\prime \prime}$ (Diercke et al. 2018), obtaining similar results. The main difference is that with a larger window the map is smoother and the values are a little smaller.

Figure 11 shows the result of the average transversal velocity of the whole temporal series with the width of the arrows being proportional to the magnitude. The velocities at photospheric levels are around $\sim 1 \mathrm{~km} \mathrm{~s}^{-1}$ while the velocity at chromospheric levels reach values of $\sim 2 \mathrm{~km} \mathrm{~s}^{-1}$. The background is the reverse image at the line core of the $\mathrm{Ca}$ II line, which helps to locate the position of the filament.

5 The latest version is hosted at https://gi thub.com/Hypnus1803/ FlowMapsGUI 

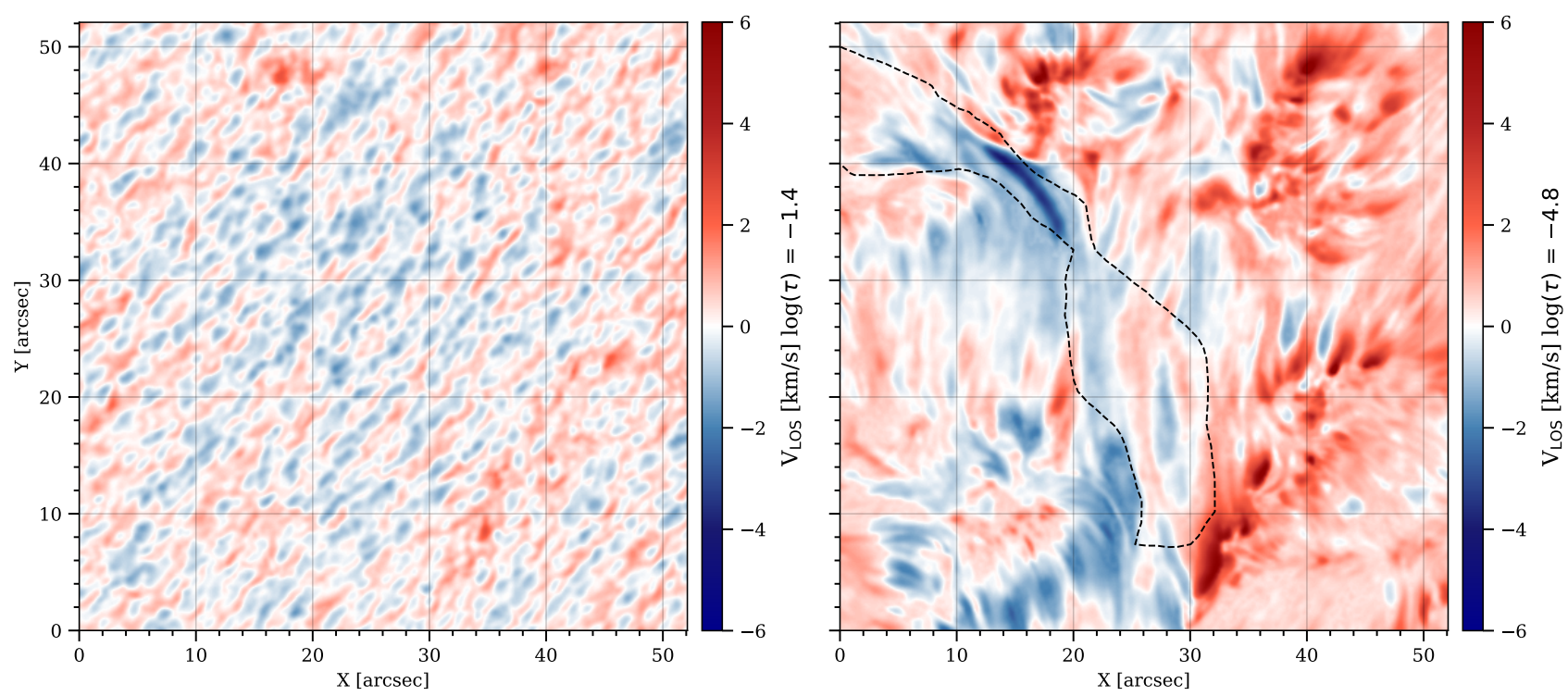

Fig. 9. Longitudinal velocity stratification inferred with NICOLE at two atmospheric layers: $\log (\tau)=-1.4($ left panel $)$ and $\log (\tau)=-4.8($ right panel). The black dashed contour draws the shape of the filament seen in $\mathrm{H} \alpha$ by CHrotel.

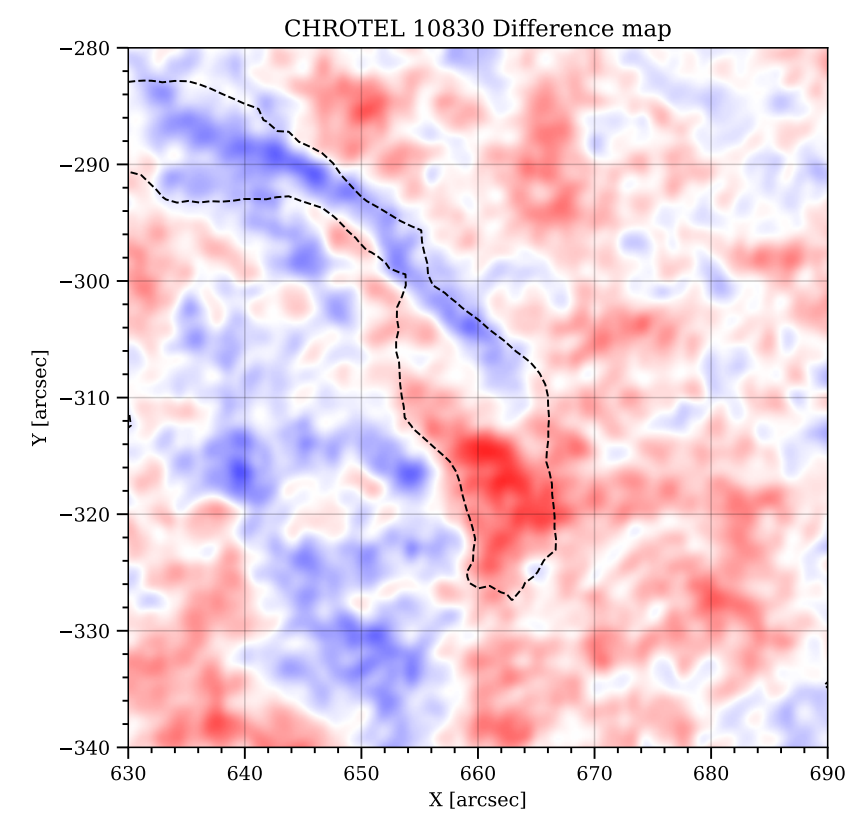

Fig. 10. Difference between the blue and red sides $( \pm 0.7 \AA)$ of the He I $10830 \AA$ with contours of $\mathrm{H} \alpha$ taken by Chrotel. As in the previous figure, blue color represent motions toward the observer. Axes are in heliocentric coordinates.

In the lower panel we detect a velocity field that seems to have the same direction as some fibrils seen in the center of the line, but the arrows are not always aligned with them. Another intriguing fact is that almost no transverse flow is found in the upper tail where we found high upflows of material, which can be explained if, for instance, the flow is constant and does not produce any change of brightness.

Another remarkable feature is given by the opposite motions on both sides of the filament. These horizontal motions reach systematic mean flow speeds of $\sim 1.5 \mathrm{~km} \mathrm{~s}^{-1}$ (averaged over $14 \mathrm{~min}$ ). While other studies such as Zirker et al. (1998) or Diercke et al. (2018) find counterflows inside the filament and they associate these dynamics with internal motions of the filament itself, our counter-motions are detected mainly out- side the filament (like in the case of Yi \& Molowny-Horas 1995) and could be associated, for instance, with the formation of the filament axis through photospheric shearing motions (van Ballegooijen \& Martens 1989). To analyze whether there are shearing motions in the photosphere, we applied the LCT method also to the photosphere using the far wings of the $\mathrm{Ca}$ II line. This result is displayed in the upper panel of Fig. 11 and it does not show any pattern that indicate these motions, but a chaotic granular pattern. A possible explanation for this nondetection could be that such motions have stopped or that our temporal range is not enough to recover these possible small velocities (perhaps below the granulation velocities).

We detect that the flows in the bright plage zones are outward, something counter-intuitive since from the NICOLE inversion the material seems to be falling at those points. One possible explanation for this effect could be that the LCT method is also sensitive to changes in brightness produced by wave propagation. Waves moving away from areas of high magnetic field and running along field lines would produce such artifacts (Bloomfield et al. 2007). This poses the question whether the opposite motions found in the filament by the LCT method may be not real, but rather changes in the intensity, indicating that the LCT method should not be safely applied to the chromosphere. Therefore, to differentiate what is actually moving, and what are propagating waves, we would need to make a more detailed study of the temporal evolution of these oscillations at different heights (Vecchio et al. 2007). Such a study would require a larger time series since $14 \mathrm{~min}$ is not enough to study them through a Fourier analysis.

\section{Supporting the filament plasma}

The general conclusion of the inversions is that we have found a filament with a lower temperature than its environment (approximately $1000 \mathrm{~K}$ colder) and a higher density (about 20 times larger). These values may vary slightly depending on the height at which we analyzed the results. Specifically, these values refer to $\log (\tau) \sim-5$. These results are consistent with the observational definition of these structures: a filament is cool chromospheric plasma overdensity embedded in the hot and low dense solar corona (Mackay et al. 2010). 

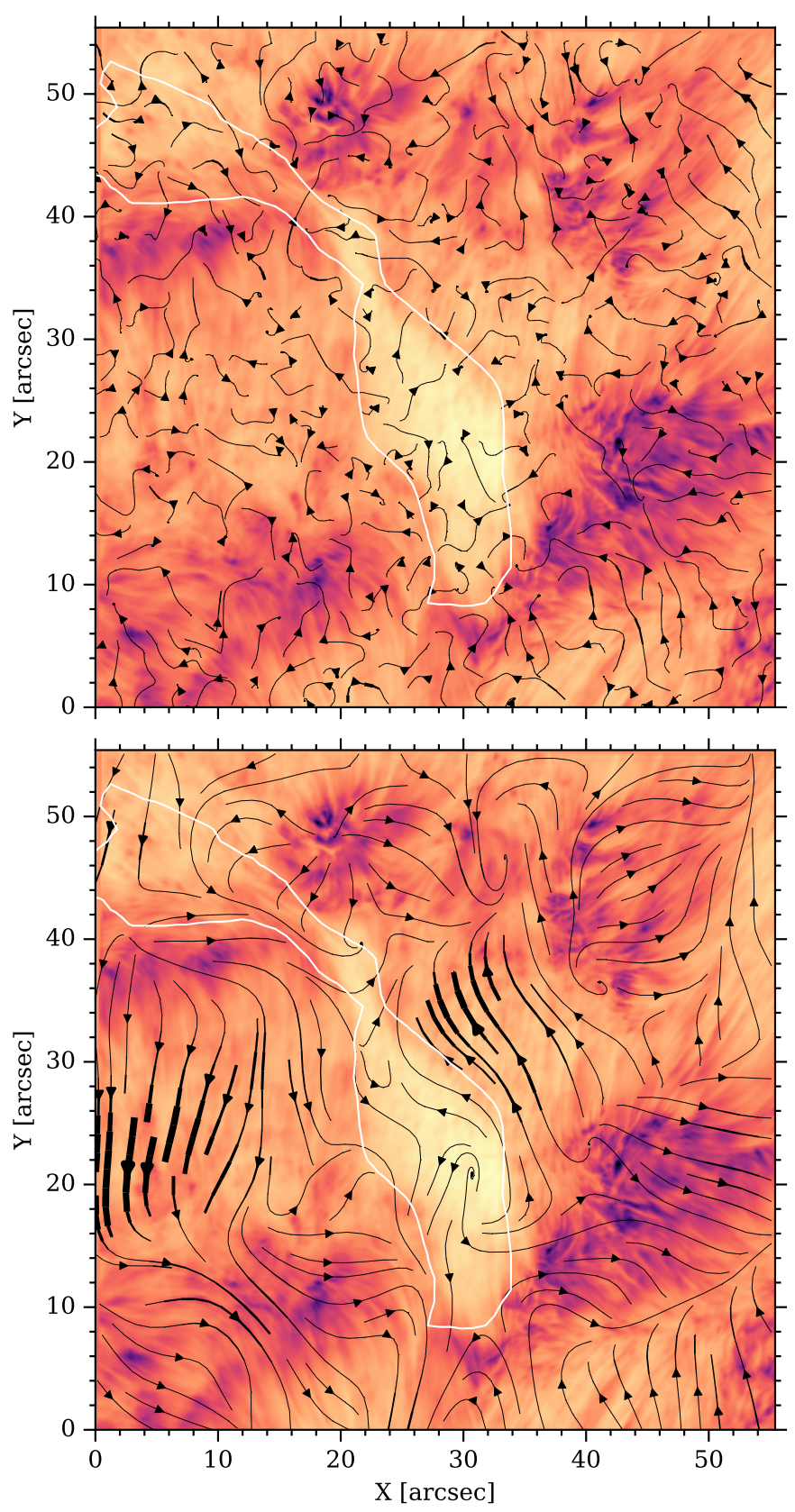

Fig. 11. Upper panel: map of the transverse component of the velocity inferred applying LCT to the temporal series at the wing of the Ca II line, tracing photospheric motions. Lower panel: same quantity calculated in the core of the $\mathrm{Ca}$ II line, tracing chromospheric layers. The width of the arrows is proportional to the magnitude.

With these properties, the filament will persist at chromospheric or coronal heights because of the magnetic field that supports the plasma against the gravitational force. The magnetic field (and in particular its topology) seems to be the main ingredient that allows this sustentation and multiple theoretical models have been proposed to explain the presence of this material for so long (Antiochos et al. 1994; van Ballegooijen \& Martens 1989; Martens \& Zwaan 2001; van Ballegooijen \& Cranmer 2010; Luna et al. 2015).

However, we note that the assumptions made for the inversions are not fully compatible with the description above. NICOLE assumes that the atmospheric models are in hydrostatic equilibrium. This implies that the gas pressure is computed from the temperature optical depth scale, neglecting the Lorentz (magnetic) force by solving, in a plane-parallel atmosphere:

$\frac{\mathrm{d} P(z)}{\mathrm{d} z}=-\rho(z) g_{\odot}(z) \stackrel{\text { ideal gas }}{\rightarrow} \frac{\mathrm{d} P(z)}{\mathrm{d} z}=-\frac{P(z)}{T(z)}\left(\frac{\mu m_{\mathrm{H}} g_{\odot}}{k_{\mathrm{B}}}\right)$,

where $g_{\odot}$ is the solar surface acceleration, $k_{\mathrm{B}}$ the Boltzmann's constant, $\mu$ the mean molecular weight and $m_{\mathrm{H}}$ the mass of an hydrogen atom. The equilibrium is obtained between the pressure and gravity forces. A consequence of Eq. (5) is that the chromospheric material under this approximation is not supported by the magnetic field and it is only the gas pressure gradient that compensates the gravitational force.

An obvious question is then whether the filament is really supported against gravity by the hydrostatic equilibrium or not. This is a reasonable question given that the model is capable of reproducing the spectral profile of the $\mathrm{Ca}$ II line. To understand how NICOLE is able to find such a solution, it is more convenient to discuss it in terms of geometric height. If we transform the stratification from optical depth to geometric height, we would have our filament at a height of $1500 \mathrm{~km}$. At this height, the pressure can still support a material with these properties. However if this overdensity is at higher heights where pressure has decreased some orders of magnitude, this material cannot be supported. The interface between these two layers will become unstable under any disturbance and a Rayleigh-Taylor instability can quickly develop and dilute the body of the prominence (Khomenko et al. 2014). In this case, the presence of a magnetic field is crucial to support this plasma and damp the formation of such instability.

Concerning this magnetic field, based on the inference from the spectropolarimetric data, the longitudinal component has values around $60 \mathrm{G}$ (see Sect. 3.3), while we can only give a maximum value of $250 \mathrm{G}$ to the transverse component due to the noise level in Stokes $Q$ and $U$ (see Sect. 3.4). This gives us a maximum value for the magnetic field strength on the order of $\sqrt{B_{\|}^{2}+B_{\perp}^{2}} \sim 260 \mathrm{G}$, a result still quite high compared to the values found in the literature (Gunár \& Mackay 2016).

Checking if the filament is in hydrostatic or magnetic equilibrium from observations is difficult. To shed some light on the problem, we have carried out several experiments. In the first numerical experiment, we started from the atmospheric model in HE inferred for the QS and analyze the influence on the emergent profile of modifications in the temperature and the pressure. To this end, we synthesized the profiles with NICOLE without imposing $\mathrm{HE}$ and adding some perturbations. The results are shown in Fig. 12, where the original stratification is shown as a black dashed line. Since the new material will not be in hydrostatic equilibrium and we want to study only the thermodynamical properties, we assume that a magnetic field is always acting to keep the material static, compensating the extra perturbations introduced by us.

The left panel of Fig. 12 shows an atmosphere where the pressure has been increased at the chromosphere $\operatorname{around} \log (\tau)=$ -5 (equivalent to a density increase). This scenario shows a dense filament model over a QS atmosphere. The result of the synthesis shows a Stokes I profile with less absorption, drawn as a solid red line. The increase in pressure (or density) causes an increase in the source function, mainly due to the higher increase of the upper level population, relatively to the increase of the lower level population through the radiative rates.

On the other hand, the right panel of Fig. 12 shows an atmosphere where we have decreased the temperature also in the chromosphere around $\log (\tau)=-5$. In this case, the synthesis 



Fig. 12. Stokes profiles synthesized under no-HE from the modifications of the model of QS under HE. Left column: only when the pressure (or density) is increased. Right column: when the temperature is decreased (even with an overdensity).

gives a deeper profile, similar to the one found inside the filament. As the sensitivity of the line to changes in temperature is higher than to changes in gas pressure, one could still increase the pressure (as one would expect for an overdense filament) and the line profile would be still deeper than that of the QS. The resulting stratification is very similar to the solution found in HE. This first experiment shows that an overdensity must be colder than the environment to reproduce the deepest profiles observed. But given the degeneration that exists between temperature and density (although the profile is more sensitive to changes in temperature), we can continue generating the same profile by increasing the density and decreasing the temperature. This does not allow us to find a unique solution.

We next investigated the effect of the height of the filament on the formation of the spectral line. To do this, we simulated the scenario of a filament of similar characteristics located at coronal heights. Because NICOLE has not been developed to correctly address the external part of the atmosphere where high temperatures are present (transition region and corona), we have used the RH code (Uitenbroek 2001) which includes the ingredients for that purpose.

In order to have an extended corona where to place the filament, we assumed that the filament is embedded in a QS atmo- sphere that we have taken from a snapshot from a 3D MHD simulation calculated with the BIFrost code (Gudiksen et al. 2011). This simulation (Carlsson et al. 2016) is publicly available as part of the Interface Region Imaging Spectro-graph (IRIS) mission (De Pontieu et al. 2014). To compute the QS model, we horizontally average the snapshot 541 (similar to the used by Quintero Noda et al. 2016 and de la Cruz Rodríguez et al. 2013b). As the simulation provides height (z), gas pressure $(P)$, and temperature $(T)$, we transform them into the quantities required by the RH code, which are: mass column density $(\rho)$, temperature $(T)$, electron density $\left(N_{\mathrm{e}}\right)$, LOS velocity $\left(v_{\mathrm{los}}\right)$, and microturbulent velocity $\left(v_{\text {turb }}\right)$ (for more details see Uitenbroek 2001). The synthesis from the original model is shown in Fig. 13 as a dashed black line. On top of this QS model we placed a filament of typical properties (Tziotziou \& Heinzel 2007; Mackay et al. 2010; Panesar 2014) with a temperature of $8000 \mathrm{~K}$, pressure

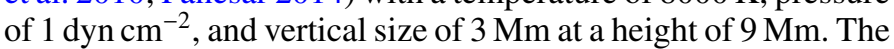
resulting Stokes $I$ profile is shown in red in the same figure.

The model shown in Fig. 13 has a pressure profile that decreases rapidly to the chromosphere $(2 \mathrm{Mm})$ and then more slowly. The temperature profile includes a smooth transition region, wider than the individual stratifications of the model due to the horizontal averaging, finally reaching a corona at 

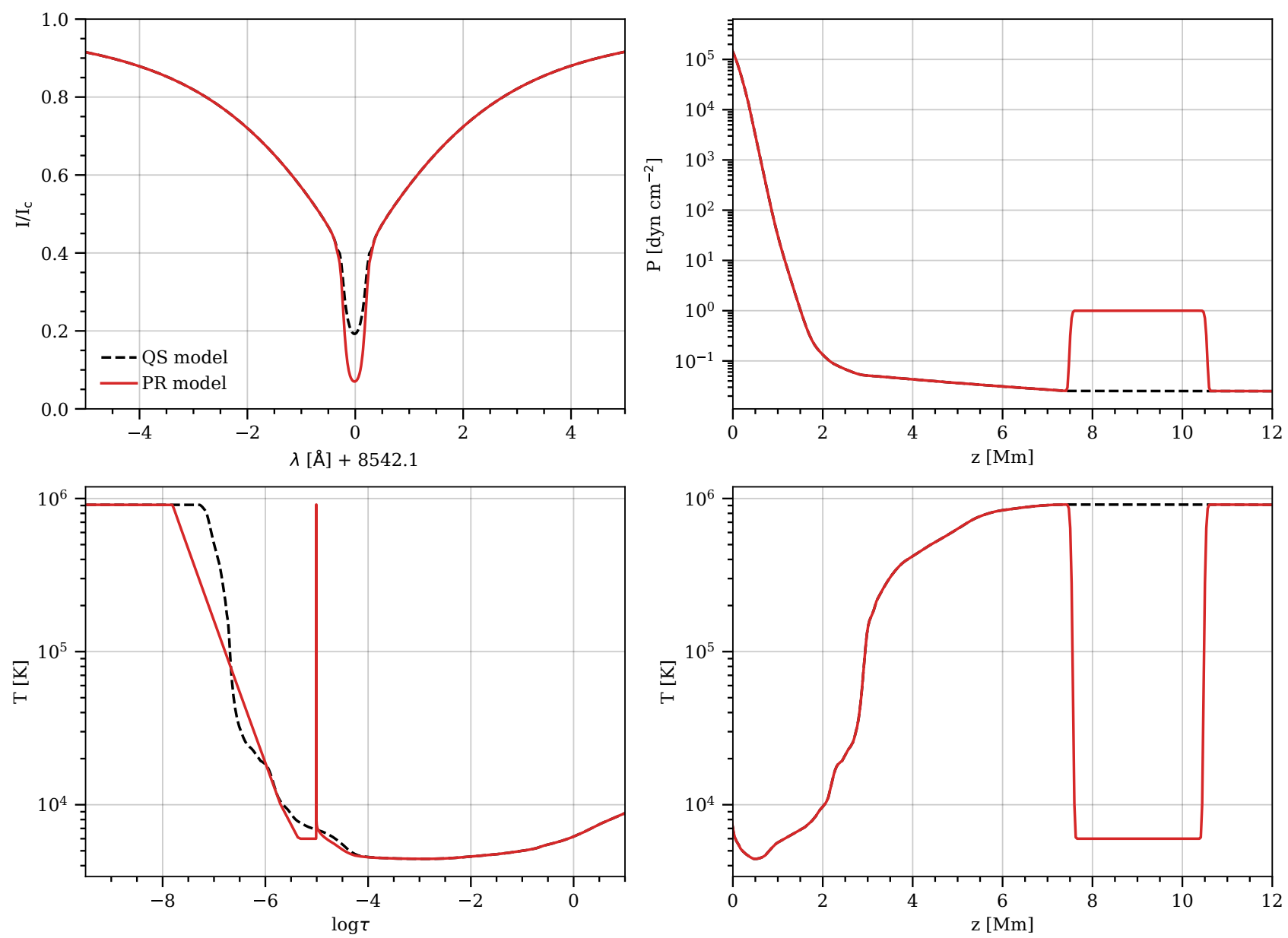

Fig. 13. Upper left panel: Stokes I profile generated from two different atmospheres, a QS model (dashed black line) and a prominence above it (red solid line). Upper right panel: gas pressure in geometrical height scale. Lower left panel: temperature in optical depth scale. Lower right panel: temperature in geometrical height scale.

$10^{6} \mathrm{~K}$. The upper left panel shows the synthesis of Stokes $I$ from both models: the QS model and the filament model. This demonstrates that a deeper Stokes profile (similar to the one observed) can be generated with a filament at coronal heights under no hydrostatic equilibrium conditions.

On the other hand, another important conclusion can be drawn in the transformation from geometric scale to optical depth scale. The lower left panel of Fig. 13 shows that the filament, physically located at coronal heights, is at the same optical depths as the original chromosphere, that is, $\log (\tau) \sim-5$. The reason of that is the small opacity of the material between the filament and the upper chromosphere that contributes almost nothing to the optical depth. This is an indication that the line is really sensitive to the filament and its conditions, independently of its height. The latter is reasonable because the filament is clearly visible in the core of the line.

Summarizing, the Ca II $8542 \AA$ spectral line is sensitive to the atmospheric conditions of the filament. However, due to the degeneration that exists among height, temperature, and pressure, we cannot give an accurate estimation of these three parameters. Other chromospheric lines with different sensitivity to temperature and pressure, such as Ca I $4227 \AA$, may be suitable for restricting the possibilities. To infer the height we should observe the filament from another perspective (e.g. in the limb) or by triangulation (e.g., using STEREO, Orozco Suárez et al. 2014; Schad et al. 2016).

\section{Summary and conclusions}

We have used the NICOLE code to study a solar filament observed on the Ca II $8542 \AA$ line. Through a careful data reduction and inversion we have studied the stratification of temperature, density and magnetic field of a region containing a filament. Thanks to the high sensitivity of the line we have inferred the atmospheric conditions from the photosphere to the chromosphere, that is, $\log (\tau) \sim[0,-5.5]$. From this inference we find that the filament appears as an overdensity (20 times greater than the environment) and with colder temperatures (about $1000 \mathrm{~K}$ lower than the environment). These results are in accordance with existing literature.

However, NICOLE assumes hydrostatic equilibrium during the inversion process. If this condition is not fulfilled in the Sun, we show that the degeneracy between temperature and pressure (or density) in the formation of the intensity profile makes it impossible to estimate these values accurately. We also show that we are insensitive to the actual height of the filament, as the small opacity of the material between the filament and the upper chromosphere contributes almost nothing to the optical depth. Therefore, even being sensitive to the "local" filament conditions, it is not possible to use this spectral line alone to distinguish between an hydrostatically supported filament at chromospheric heights and a colder and denser filament at coronal heights.

Concerning the magnetic field, we detect homogeneous fields with a strength smaller than $260 \mathrm{G}$ throughout the region 
without a clear distinct value on of the filament. Weaker fields do exist in the area, but we are not able to detect them due to the noise level in the observations. These values indicate that we are in a field regime in which the Hanle effect begins to play an important role and should be taken into account (Manso Sainz \& Trujillo Bueno 2010).

Although the filament is stable during the observations, our observations reveal gas motions operating at very small scales. From a global point of view, our filament appears to be attached to a larger filament, through which material could be transferred. We detect it as a blueshifted flow in the tail of the observed filament. From a local point of view, we have detected, using the He I $10830 \AA$ line, higher velocities of material falling from the filament than those inferred from the $\mathrm{Ca}$ II line. This material loss due to the neutral nature of $\mathrm{He}_{\mathrm{I}}$ (not sensitive to the magnetic field) is expected. These differences in velocity reminds us that the filament is not only supported by the gas pressure.

Finally, we have also studied the time series using LCT. As a result, we obtain flows practically aligned with the fibrils but with directions opposite to the velocities obtained by the Doppler-shift of the line. One possible explanation would be the propagation of waves emerging from the magnetic field concentrations of the plage and could serve as a diagnosis of the field topology. However, to confirm this, further work remains is necessary.

Acknowledgements. We would like to thank the anonymous referee for thei comments and suggestions. The authors also wish to thank Ivan Milić for useful discussions during the analysis of the presented work. Financial support by the Spanish Ministry of Economy and Competitiveness through projects AYA2014 60476-P and AYA2014-60833-P are gratefully acknowledged. CJDB acknowledges Fundación La Caixa for the financial support received in the form of a $\mathrm{PhD}$ contract. AAR also acknowledges financial support through the Ramón y Cajal fellowships. This research has made use of NASA's Astrophysics Data System Bibliographic Services. Jd1CR is supported by grants from the Swedish Research Council (2015-03994), the Swedish National Space Board (128/15) and the Swedish Civil Contingencies Agency (MSB). This project has received funding from the European Research Council (ERC) under the European Union's Horizon 2020 research and innovation program (SUNMAG, grant agreement 759548). The Swedish 1-m Solar Telescope is operated on the island of La Palma by the Institute for Solar Physics of Stockholm University in the Spanish Observatorio del Roque de los Muchachos of the Instituto de Astrofísica de Canarias. The Institute for Solar Physics is supported by a grant for research infrastructures of national importance from the Swedish Research Council (registration number 2017-00625). This research has made use of NASA's Astrophysics Data System Bibliographic Services. We acknowledge the community effort devoted to the development of the following open-source packages that were used in this work: numpy (http://www. numpy.org/), matplotlib (https: //matplotlib.org/) This research has made use of SunPy, an open-source and free community-developed solar data analysis package written in Python (SunPy Community et al. 2015). The author thankfully acknowledges the technical expertise and assistance provided by the Spanish Supercomputing Network (Red Española de Supercomputación), as well as the computer resources used: the LaPalma Supercomputer, located at the Instituto de Astrofísica de Canarias.

\section{References}

Antiochos, S. K., Dahlburg, R. B., \& Klimchuk, J. A. 1994, ApJ, 420, L41 Asensio Ramos, A., de la Cruz Rodríguez, J., Martínez González, M. J., \& SocasNavarro, H. 2017, A\&A, 599, A133

Aulanier, G., \& Démoulin, P. 2003, A\&A, 402, 769

Bethge, C., Peter, H., Kentischer, T. J., et al. 2011, A\&A, 534, A105

Bloomfield, D. S., Lagg, A., \& Solanki, S. K. 2007, ApJ, 671, 1005

Campos Rozo, J. I., \& Vargas Domínguez, S. 2015, AGU Fall Meeting Abstracts

Carlin, E. S., Asensio Ramos, A., \& Trujillo Bueno, J. 2013, ApJ, 764, 40

Carlsson, M., Hansteen, V. H., Gudiksen, B. V., Leenaarts, J., \& De Pontieu, B. 2016, A\&A, 585, A4

Casini, R., López Ariste, A., Tomczyk, S., \& Lites, B. W. 2003, ApJ, 598, L67 Cheung, M. C. M., Schüssler, M., \& Moreno-Insertis, F. 2007, A\&A, 461, 1163 Cox, A. N. 2000, Allen's Astrophysical Quantities (New York: Springer-Verlag), 700 de la Cruz Rodríguez, J., \& Piskunov, N. 2013, ApJ, 764, 33

de la Cruz Rodríguez, J., Socas-Navarro, H., Carlsson, M., \& Leenaarts, J. 2012, A\&A, 543, A34

de la Cruz Rodríguez, J., Rouppe van der Voort, L., Socas-Navarro, H., \& van Noort, M. 2013a, A\&A, 556, A115

de la Cruz Rodríguez, J., De Pontieu, B., Carlsson, M., \& Rouppe van der Voort, L. H. M. 2013b, ApJ, 764, L11

de la Cruz Rodríguez, J., Hansteen, V., Bellot-Rubio, L., \& Ortiz, A. 2015a, ApJ, 810,145

de la Cruz Rodríguez, J., Löfdahl, M. G., Sütterlin, P., Hillberg, T., \& Rouppe van der Voort, L. 2015b, A\&A, 573, A40

De Pontieu, B., Title, A. M., Lemen, J. R., et al. 2014, Sol. Phys., 289, 2733

Diercke, A., Kuckein, C., Verma, M., \& Denker, C. 2018, A\&A, 611, A64

Gudiksen, B. V., Carlsson, M., Hansteen, V. H., et al. 2011, A\&A, 531, A154

Gunár, S., \& Mackay, D. H. 2016, A\&A, 592, A60

Heinzel, P. 2007, ASP Conf. Ser., 368, 271

Hill, F., Fischer, G., Grier, J., et al. 1994, Sol. Phys., 152, 321

Keppens, R., \& Xia, C. 2014, ApJ, 789, 22

Khomenko, E., Díaz, A., de Vicente, A., Collados, M., \& Luna, M. 2014, A\&A, 565, A45

Khomenko, E., Collados, M., \& Díaz, A. J. 2016, ApJ, 823, 132

Kippenhahn, R., \& Schlüter, A. 1957, Z. Astrophys., 43, 36

Kuckein, C., Martínez Pillet, V., \& Centeno, R. 2012, A\&A, 539, A131

Kuckein, C., Verma, M., \& Denker, C. 2016, A\&A, 589, A84

Leenaarts, J., \& Carlsson, M. 2009, ASP Conf. Ser., 415, 87

Leenaarts, J., de la Cruz Rodríguez, J., Kochukhov, O., \& Carlsson, M. 2014 ApJ, 784, L17

Lemen, J. R., Title, A. M., Akin, D. J., et al. 2012, Sol. Phys., 275, 17

Leroy, J. L., Ratier, G., \& Bommier, V. 1977, A\&A, 54, 811

Lin, Y., Engvold, O. R., \& Wiik, J. E. 2003, Sol. Phys., 216, 109

Lites, B. W. 2005, ApJ, 622, 1275

López Ariste, A., \& Casini, R. 2002, ApJ, 575, 529

Luna, M., Moreno-Insertis, F., \& Priest, E. 2015, ApJ, 808, L23

Mackay, D. H., Karpen, J. T., Ballester, J. L., Schmieder, B., \& Aulanier, G. 2010, Space Sci. Rev., 151, 333

Manso Sainz, R., \& Trujillo Bueno, J. 2010, ApJ, 722, 1416

Martens, P. C., \& Zwaan, C. 2001, ApJ, 558, 872

Martínez González, M. J., Manso Sainz, R., Asensio Ramos, A., \& Belluzzi, L. 2012, MNRAS, 419, 153

Martínez González, M. J., Manso Sainz, R., Asensio Ramos, A., et al. 2015, ApJ, 802, 3

Neckel, H., \& Labs, D. 1984, Sol. Phys., 90, 205

Okamoto, T. J., Liu, W., \& Tsuneta, S. 2016, ApJ, 831, 126

Orozco Suárez, D., Asensio Ramos, A., \& Trujillo Bueno, J. 2014, A\&A, 566, A46

Panesar, N. K. 2014, PhD Thesis, Georg-August-Universität Göttingen, Institut für Astrophysik, Germany

Park, H., Chae, J., Song, D., et al. 2013, Sol. Phys., 288, 105

Pesnell, W. D., Thompson, B. J., \& Chamberlin, P. C. 2012, Sol. Phys., 275, 3

Quintero Noda, C., Shimizu, T., de la Cruz Rodríguez, J., et al. 2016, MNRAS, 459, 3363

Ruiz Cobo, B., \& del Toro Iniesta, J. C. 1992, ApJ, 398, 375

Schad, T. A., Penn, M. J., Lin, H., \& Judge, P. G. 2016, ApJ, 833, 5

Scharmer, G. B., Bjelksjo, K., Korhonen, T. K., Lindberg, B., \& Petterson, B.

2003, Proc. SPIE, 4853, 341

Scharmer, G. B., Narayan, G., Hillberg, T., et al. 2008, ApJ, 689, L69

Schmieder, B., Tziotziou, K., \& Heinzel, P. 2003, A\&A, 401, 361

Socas-Navarro, H. 2005, ApJ, 633, L57

Socas-Navarro, H., Trujillo Bueno, J., \& Ruiz Cobo, B. 2000, ApJ, 530, 977

Socas-Navarro, H., de la Cruz Rodríguez, J., Asensio Ramos, A., Trujillo Bueno,

J., \& Ruiz Cobo, B. 2015, A\&A, 577, A7

Stellmacher, G., Wiehr, E., \& Dammasch, I. E. 2003, Sol. Phys., 217, 133

Štěpán, J., \& Trujillo Bueno, J. 2016, ApJ, 826, L10

SunPy Community, Mumford, S. J., Christe, S., et al. 2015, Comput. Sci. Discov., 8, 014009

Tziotziou, K., \& Heinzel, P. 2007, ASP Conf. Ser., 368, 217

Uitenbroek, H. 1989, A\&A, 213, 360

Uitenbroek, H. 2001, ApJ, 557, 389

van Ballegooijen, A. A., \& Cranmer, S. R. 2010, ApJ, 711, 164

van Ballegooijen, A. A., \& Martens, P. C. H. 1989, ApJ, 343, 971

van Noort, M., Rouppe van der Voort, L., \& Löfdahl, M. G. 2005, Sol. Phys., 228, 191

Vecchio, A., Cauzzi, G., Reardon, K. P., Janssen, K., \& Rimmele, T. 2007, A\&A, 461, L1

Wedemeyer-Böhm, S., \& Carlsson, M. 2011, A\&A, 528, A1

Yi, Z., \& Molowny-Horas, R. 1995, A\&A, 295, 199

Zirker, J. B., Engvold, O., \& Martin, S. F. 1998, Nature, 396, 440 